\title{
A Spectral Mapping Theorem for Representations of Compact Groups
}

\author{
W: Areñd and C. D'Antoni ${ }^{1}$ )
}

Sei $U$ eine stark stetige beschränkte Darstellung einer lokal kompakten Gruppe $G$ auf einem Banachraum $E$. Ist $\mu$ ein beschränktes reguläres Borelma $\beta$, so bezeichnen wir mit $U(\mu)$.den Operator $U(\mu)=\int U(t) d \mu(t)$. Wenn $G$ abelsch ist, so ist bekannt, daß

$$
\sigma(U(\mu))=\ddot{\mu}(\operatorname{sp}(U))^{-}
$$

wenn der singuläre stetige Anteil von $\mu$ Null ist (wobei $\sigma(U(\mu)$ ) das Spektrum des Operators $U(\mu), \mathrm{sp}(U)$ das Arveson-Spektrum von $U$ und $\hat{\mu}$ die Fourier-Stieltjes Transformierte von $\mu$ bezeichnet).

Im vorliegenden Artikel wird ein entsprechender spektraler Abbildungssatz für kompakte (nicht-abelsche) Gruppen und absolut stetige Maße bewiesen. Ferner wird gezeigt, da B - im Gegensatz zum abelschen Fall - der spektrale Abbildungssatz für atomáre Maße nicht gilt.

Пусть $U$ сильно непрерывное ограниченное представление локально компактой группы $G$ на банаховое пространство. Если $\mu$ ограниченная регулярная борелевская мера, пусть $U(\mu)$ обовначаѐт оператор $U(\mu)=\int U(t) d \mu(t)$. Если $G$-аб̆елевая, то известно, что

$$
\sigma(U(\mu))=\hat{\mu}(\operatorname{sp}(U))^{-}
$$

если сингујярная непрерывная часть $\mu$ равна нулю (гле $\sigma(U(\mu))$ обозначает спектр оператора $U(\mu)$, sp $(U)$-арвесонскип̆ спектр $U$, и $\hat{\mu}$-фурье-стильтьесовое преобразование $\mu$ ).

В данной статье доказана подобная спектральная теорема об отображенин для компактных (не абелевых) групп и абсолютно непрерывных мер. Кроме того доказано, что в противоположность абелевому случаю для чисто прерывных мер спектральная теорема об отображении не верна.

Let $U$ be a strongly continuous bounded representation of a locally compact group $G$ on a Banach space $E$. For a bounded regular Borel measure $\mu$ on $G$, we denote by $U(\mu)$ the operator i , $U(\mu)=\int U(t) d \mu(t)$. If $G$ is abelian, it is known that

$$
\sigma(U(\mu))=\hat{\mu}(\operatorname{sp} U)^{-}
$$

holds if the continuous singular part of $\mu$ is zero (where $\sigma(U(\mu)$ ) denotes the spectrum of the operator $U(\mu), \operatorname{sp}(U)$ the Arveson-spectrum of $U$ and $\hat{\mu}$ the Fourier-Stieltjes transformation of $\mu$.)

In the present article a corresponding spectral mapping theorem is proved for compact (non: abelian) groups and absolutely continuous measures. Moreover, it is shown that - in contrary to the abelian case - the spectral mapping theorem fails for purely discontinuous measures.

1) Supported in part by the Italian C.N.R. 


\section{Introduction}

Given a suitably continuous homomorphism $U$ of a locally compact group $G$ into the group of all isometries on a Banach space $E$, it is possible to define the representation of $M(G)$, the Banach algebra of all bounded regular Borel measures on $G$, by

$$
U(\mu)=\int U_{t} d \mu(t)
$$

If $G$ is abelian (resp. compact) in the theory of spectral subspaces the spectrum sp ( $U$ ) of $U$ is defined as a certain subset of the dual group of $G$ [2] (resp. the dual object of $G[6])$.

In the abelian case a spectral mapping theorem is proved in [5] (see also [9]), stating

$$
\sigma(\dot{U}(\mu))=\hat{\mu}\left(\operatorname{sp}^{\prime}(U)\right)^{-}
$$

for every measure $\mu \in M(G)$ whose continuous part belongs to $\dot{L}^{1}(G)$. The purpose of this paper is to prove the analogue of this theorem for compact groups.

It is interesting that the corresponding theorem does not hold in this generality for the non-abelian case: In fact, a counterexample given in this paper shows that it may fail to hold for purely discontinuous measures.

\section{The main theorem}

Let $E$ be a Banach space, $G$ a compact group and $U$ a homomorphism of $G$ into the group of invertible operators on $E$. That means:

$U_{t} \in \mathscr{L}(E), U_{s t}=U_{s} U_{t}, U_{e}=I$ for $s, t \in G$, where $e \in G$ is the unit of $G$ and $I$ the identity operator on $E$.

Suppose $U$ is continuous in the sense of [2: assumption 1.1]. In particular, $U$ may be strongly continuous. Then for $\mu \in M(G)$ the operator $U(\mu)$ can be defined by

$$
U(\mu)=\int U_{t} d \mu(t)
$$

(see [2]). $U(\mu)$ is a bounded operator on $E,\|U(\mu)\| \leqq c\|\mu\|$ for a positive constant $c$ and all $\mu \in M(G)$. For $\mu, \nu \in M(G)$ we have $U(\mu * v)=U(\mu) U(v)$, and $U_{t}=U\left(\delta_{t}\right)$ for the Dirac measure $\delta_{t}$ at the point $t \in G$. Thus $U$ extends to a representation of $M(G)$, on $E$.

Denote by $G$ the dual object of $G$, i.e. $G$ is the set of all equivalence classes of continuous unitary irreducible representations of $G^{\circ}$. For $\alpha \in \hat{G}$ chose $u_{\alpha} \in \alpha . u_{\alpha}$ is a homomorphism of $G$ into the group of all unitary $n_{a} \times n_{a}$-matrices. Denote by $u_{a i j}(t)$ the matrix entries of $u_{a}(t)$, and by $u_{\alpha i j}$ the coordinate function $t \rightarrow u_{\alpha i j}(t)$ on $G(1 \leqq i$, $\left.j \leqq n_{\alpha}\right)$. For $\mu \in M(G)$ let $\mu_{\alpha}=\int u_{\alpha}\left(t^{-1}\right) d \mu(t)$ for $\alpha \in \hat{G}$,(i.e. $\hat{a}_{\alpha}$ is the $n_{\alpha} \times n_{\alpha}$-matrix. $\left.\left(\int u_{a i j}\left(t^{-1}\right) d \mu(t)\right)_{i j}\right)$. We identify $L^{i}(G)$ with a subspace of $M(G)$ in the canonical way. Thus we set $\hat{f}_{\dot{\alpha}}=(f m)_{\hat{\alpha}}(\alpha \in \hat{G})$ and $U(f):=U(f m)$ for $f \in L^{1}(G)$, where $m$ denotes the Haar measure on $G$.

2.1 Definition: $\operatorname{sp}(U)=\left\{\alpha \in \hat{G} \mid \hat{f}_{\alpha}=0\right.$ for all $\left.f \in I\right\}$, where $I$ is the closed ideal of $L^{1}(G)$ defined by $I=\left\{f \in L^{1}(G) \mid U(f)={ }^{\prime} 0\right\}$.

This definition is equivalent to the one given in [6] and coincides with Arveson's definition in the abelian case. We can now formulate the main theorem. 
2.2 Theorem: 1. For $\mu \in M(\dot{G}), \operatorname{Po}(U(\mu))=\underset{\alpha \in \operatorname{sp}(U)}{\bigcup} \sigma\left(\mu_{a}\right)$.

2. For $f \in L^{1}(G)$,

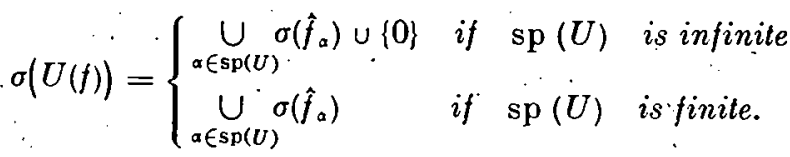

Note: $\sigma(T)$ denotes the spectrum and $P \sigma(T)$ the point spectrum of a bounded operator $T$ on $E$. For $\mu \in M(G), \alpha \in G$ let $\sigma\left(\beta_{\alpha}\right)$ denote the set of all eigenvalues of the matrix $\mu_{\dot{\alpha}}$.

For the proof of the theorem we need some lemmas.

The coordinate functions are continuous and satisfy

$$
\begin{aligned}
& u_{\alpha i j} * u_{\beta k l}=1 / n_{a} \delta_{\alpha \beta} \delta_{j k} u_{\alpha i l} \\
& \left(\alpha ; \beta \in \hat{G}, 1 \leqq i, j \leqq n_{\alpha}, \quad 1 \leqq k, l \leqq n_{\beta}\right) \quad \text { (see [8:27.20]). }
\end{aligned}
$$

Let $V_{\alpha i j}:=U\left(n_{\alpha} u_{a i j}\right) \quad\left(\alpha \in \hat{G}, 1 \leqq i, j \leqq n_{\alpha}\right)$.

The operators $V_{a i j}$ satisfy the composition rules

$$
\left.\begin{array}{l}
V_{a i j} V_{\beta k l}=0 \text { if } \alpha \neq \beta \text { or } j \neq k, \ldots \\
V_{a i j} V_{a j l}=V_{a i l} .
\end{array}\right\}
$$

In particular $V_{\alpha i i}$ and $P_{\alpha}:=\sum_{i=1}^{n} V_{\alpha i i}$ are projections $\left(\alpha \in \hat{G}, 1 \leqq i . \leqq n_{\alpha}\right)$ :

2.3 Lemma: For $\mu \in M(G), \alpha \in \hat{G}$,

a) $P_{\alpha} U(\mu) \doteq U(\mu) P_{\alpha}=\sum_{i, j=1 \ldots n_{\alpha}} a_{a j i} V_{a i j} \quad\left(1 \leqq i, j \leqq n_{\alpha}\right)$

b) $V_{a i j} P_{a}=P_{a} V_{a i j}=V_{a i j} \quad\left(1 \leqq i, j \leqq n_{a}\right)$,

c) $P_{\alpha} P_{\beta}=P_{\beta} P_{a}=0$ for $\alpha \neq \beta$.

Proof: By [8: 27.20],

$$
\mu * u_{s i i}=\sum_{j=1}^{n_{\alpha}} \int \overline{u_{a j i}(s)} d \mu(s) u_{a j i}=\sum_{j=1}^{n_{\alpha}} \hat{\mu}_{a i j} u_{\alpha i j}
$$

and

$$
u_{\alpha i i} * \mu=\sum_{j=1}^{n_{\alpha}} \cdot \int \overline{u_{\alpha i j}(s)} d \mu(s) u_{\alpha i j}=\sum_{j=1}^{n_{\alpha}} \hat{\mu}_{\alpha j i} u_{a j i} .
$$

Consequently,

$$
\begin{aligned}
P_{\alpha} U(\mu) & =U\left(n_{\alpha} \sum_{i=1}^{n_{\alpha}} u_{a i i} * \mu\right)=U\left(n_{\alpha} \sum_{i j=1}^{n_{\alpha}} a_{a j i} u_{\alpha i j}\right)=\sum_{i j=1}^{n_{\alpha}} \rho_{a j, i} V_{a i j} \\
& =U\left(\mu * n_{\alpha} \sum_{j=1}^{n_{\alpha}} u_{\alpha j i}\right)=U(\mu) P_{\alpha}
\end{aligned}
$$

b)! and c) are obvious from (C)

There is an alternative description of $\mathrm{sp}(U)$. 
$2.4 \mathrm{Lemma}: \operatorname{sp}(U)=\left\{\alpha \in G \mid P_{\alpha} \neq 0\right\}$.

Proof: Let $S=\left\{a \in \hat{G} \mid P_{\alpha} \neq 0\right\}$.

a) Let $\alpha \in \operatorname{sp}(U)$. Since $\left(u_{\alpha i i}\right)_{\alpha i j}=1 / n_{\alpha}$ (see [8:-27.19]), $u_{\alpha i i} \notin I$ by the definition of $\operatorname{sp}\left(U_{.}\right.$. From the definition of $I$ follows that $V_{a i i} \neq 0\left(1 \leqq i \leqq n_{a}\right)$ and consequently, $P_{a} \neq 0$ (use-2.3b)). Hence $\alpha \in S$.

b) Let $\alpha \in S$. Then $V_{a i j} \neq 0\left(1 \leqq i, j \leqq n_{a}\right)$. From $\left.2.3 a\right)$ and $(C)$ follows that $V_{\alpha i i} P_{\alpha} U(f) V_{a j j}=\hat{f}_{a j i} V_{a i j}$. Hence, $f \hat{\epsilon} I$ implies $\hat{f}_{\alpha}=0$; i.e. $\alpha \in \operatorname{sp}(U)$

2.5 Lemma: If $U(f) x=0$ for all $f \in L^{1}(G)$, then $x=0$.

Proof: For every $\varphi \in E_{*}$ (a separating subspace of the dual space of $E$ according to the assumption in [1] (if $U$ is strongly continuous. $E_{*}$ is just the dual space of $\left.E^{\prime}\right)$ ), $\int\left\langle U_{t} x, \varphi\right\rangle f(t) d t=0$ for every $f \epsilon^{\prime} L^{1}(G)$ by hypothesis. Since the function $t \rightarrow\left\langle U_{t} x, \varphi\right\rangle$ is continuous, it is identically zero, hence $\langle x, \varphi\rangle=0$. This implies $x=0, E_{*}$ being separating

2.6 Lemma: If $P_{a} x=0$ for every $\alpha \in \operatorname{sp}(U)$, then $x=0$.

Proof: The hypothesis implies thàt $P_{\alpha} x=0$ for every, $\alpha \in \hat{G}$. Consequently, $U\left(u_{a i j}\right) x=0$ for every $\alpha \in \hat{G}, i, j \in\left\{1, \ldots, n_{\alpha}\right\}$, hence $U(p) x=0$ for every trigonometric polynomial $\dot{p}$ on $G$, which implies $U(f) x=0$ for every $f \in L^{\mathbf{1}}(G)$, the trigonometric polynomials being dense in $L^{1}(G)$. It follows from 2.5 that $x=0$.

2.7 Lemma: If $\beta_{\alpha} \doteq 0$ for every $\alpha \in \operatorname{sp}^{\prime}(U)$, then $U(\mu)=0$.

Proof: If $\mu_{\alpha}=0$ for every $\alpha \in \operatorname{sp}(U)$, then $P_{\alpha} U(\mu)=0$ for every $\alpha \in \operatorname{sp}(U)$ by 2.3 ; consequently $U(\mu)=0$ by 2.6

Proof of the theorem:

1. Let $\mu \in M(G)$. For $\alpha \in \operatorname{sp}\left(U_{1}\right)$ let $F=P_{\mathrm{a}} E$. $F$ is not reduced to 0 and invariant under $U(\mu)$ by 2.3. Denote by $U_{a}$ the restriction of $U(\mu)$ to $F$. $V_{a i i}$ is a projection which leaves $F$ invariant by 2.3 Set $F_{i}:=V_{a i i} F\left(1 \leqq i \leqq n, n:=n_{a}\right)$. We have $F=F_{1}$ it $\ldots+F_{n}$ and $F_{i} \cap F_{j}=0$ for $i \neq j$. From the composition rules (C) it can be seen that the restriction of $V_{\text {aij }}$. to $F_{i}$ is an isomorphism of $F_{i}$ onto $F_{j}$, which we denote by $V_{i j}$. Moreover, $V_{i}=I_{i}$ (the identity operator on $\left.F_{i}\right)$,

$$
\left(\nabla_{i j}\right)^{-1}=V_{j i} \quad(1 \leqq i, j \leqq n) \text {. }
$$

For $x=x_{1}+\cdots+x_{n} \in F_{1}+\cdots+F_{n}=F$ we have by 2.3 and (C)

$$
U(\mu) x=U(\mu) P_{a} x=\sum_{i, j=1}^{n} a_{a i j} V_{a j i} x=\sum_{i, j=1}^{n} a_{a i j} V_{j i} x_{i}
$$

Set $H:=\prod_{m=1}^{n} F_{1}$ (n-times the Cartesian product of $F_{1}$ with any norm inducing the product topology on $H)$ and define $V: F \rightarrow H$ by $\left(x_{1}+\cdots+x_{n}\right) \rightarrow\left(V_{11} x_{1}, V_{12} x_{2}, \ldots\right.$, $\left.V_{1 n} x_{n}\right) . V$ is an isomorphism with inverse

$$
V^{-1}: H \rightarrow F,\left(y_{1}, \ldots ; y_{n}\right) \rightarrow\left(V_{11} y_{1}+\dot{V}_{21} y_{2}+\cdots+V_{n 1} y_{n}\right)
$$

Let $\hat{U}_{a}=\dot{V} U_{a}^{\prime} V^{-1}$. From (1) it can be seen that $\hat{U}_{a}$ has the matrix representation

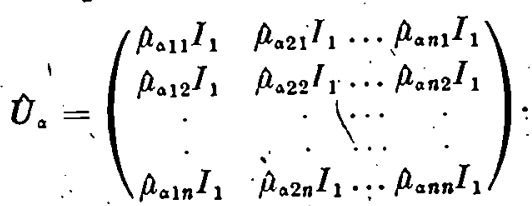


From this it is easy to sec that $\sigma\left(U_{\alpha}\right)=\sigma\left(\hat{U}_{a}\right)=\sigma\left(\hat{A}_{\alpha}\right)$ and $\sigma\left(U_{\alpha}\right)=P \sigma\left(U_{\alpha}\right)$. Thus' $\underset{f \operatorname{sp}(U)}{\dot{V}} \sigma\left(\hat{\mu}_{\dot{\alpha}}\right) \subset P \sigma(U(\mu))$.

To.show the inverse inclusion, let $\lambda \in P \sigma U((\mu))$. Then there exists $x \in E$ such that $x \neq 0$ and $U(\mu) x=\lambda x$. By 2.6 there is an $\alpha \in \operatorname{sp}(U)$ such that $y=P_{\alpha} x \neq 0$. It follows. from 2.3 that $U(\mu) y=\lambda y$. Hence $\lambda \in \sigma\left(U_{a}\right)=\sigma\left(\mu_{a}\right)$.

2. Let $f \in L^{1}(G)$. a) If $\operatorname{sp}(U)$ is infinite, $0 \in \underset{\alpha \in \operatorname{sp}(U)}{\bigcup_{0\left(f_{a}\right)}}$, because $\left(\|_{!} \hat{f}_{a} !\right)_{\alpha \in G}$ tends to 0 at $\infty$ (in the discrete topology on $G$ ). Hence $0 \in \sigma(U(f))$ by 1 .

We show that $\sigma(U(f)) \backslash\{0\} \subset \cup \sigma\left(\hat{f}_{\alpha}\right)$. Let $\lambda \notin \cup \sigma\left(\hat{f}_{\alpha}\right) \cup\{0\}$. It has to be shown that $\lambda \notin \sigma(U(f))$. Since $\left(\left\|\hat{f}_{a}\right\|\right)_{\alpha \in G}$ tends to zero at $\infty \in \operatorname{sp}$, the set $N:=\left\{\alpha \in \cdot \dot{G} \mid \lambda \in \dot{\sigma}\left(\hat{f}_{\alpha}\right)\right\}$ is finite. There exists a trigonometric polynomial $g$ on $G$ such that $\hat{g}_{a}=f_{a}$ for all $\alpha \in N$ and $\hat{g}_{a}=0$ for $\alpha \notin N$ (use $\left(u_{\alpha i j}\right)_{\beta k l}=\left(u_{a i j} * u_{\beta k l}\right)(e)=1 / n_{a} \delta_{a \beta} \delta_{i l} \delta_{k j}$ by [8:27.20 (iii)]). Let $h=f-g$. Then $h \in L^{1}(G), \hat{h}_{\alpha}=\hat{f}_{\alpha}$ for $\alpha \notin N$ and $\hat{h}_{\alpha}=0$ for $\alpha \in N$. In particular, $\lambda \notin \bigcup_{a \in Q^{\circ}} \sigma\left(\hat{h}_{a}\right)$; which implies by $[1: 4.3]$ that $\lambda \notin \sigma_{L^{2}(G)}(h)$, i.e. there exists $k \in L^{1}(G)$ such that $\left(\lambda \delta_{e}-h\right) *\left(1 / \lambda \delta_{e}-k\right)=\left(1 / \lambda \delta_{e}-k\right) *\left(\lambda \delta_{e}-h\right)=\delta_{e}$. Hence

$$
(\lambda-U(h))(1 / \lambda-\dot{U}(k))=(1 / \lambda-U(k))(\lambda-U(h))=. \dot{I}
$$

From our assumption on $\lambda ; N \cap \operatorname{sp}(U)=\varnothing$, hence $\hat{h}_{\mathrm{a}}=f_{\alpha}$ for all $\alpha \in \operatorname{sp}(U)$. This implies that $U(h)=O(f)$ by 2.7. Consequently $(\lambda-U(f))$ is invertible in $\mathscr{L}(E)$ by (2), i.e. $\lambda \notin \sigma(U(f))$.

b) Let sp $(U)$ be finité and suppose $0 \notin \bigcup_{\alpha \in \operatorname{sp}(U)} \sigma\left(\hat{f}_{\alpha}\right)$. In order to show that $0 \notin \sigma(U(f))$, take a trigonometric polynomial $g$ satisfying $\hat{g}_{a}=\left(\hat{f}_{a}\right)^{-1}$ for all $\alpha \in \mathbf{s p}(U)$. Then $(g * f)_{\hat{\alpha}}=\hat{g}_{\alpha} f_{\alpha}=I=\left(\delta_{e}\right)=(f * g)_{\alpha}^{\hat{\alpha}}$ for every $\alpha \in \operatorname{sp}(U)$. It follows from $(2.7)$ that $U(f) U(g) \stackrel{U}{=} U(g) U(f)=I$, hence of $0 \notin \sigma(U,(f))$

\section{Discrete measures}

It has been shown in [5] that for abelian groups the theorem corresponding to the second part of 2.2 remains true if $f$ is replaced by a measure on $G$ whose singular part is completely discontinuous. This is no longer true for compact non-abelian groups. In fact, while we can prove that theorem 2.2 part 2 holds for point measures, we show that there exists a completely discontinuous measure $\mu$ on a compact group $G$ such that

$$
\sigma(U(\mu)) \neq \overline{\alpha \in \operatorname{sp}(U)} \sigma\left(\mu_{\alpha}\right),
$$

where $U$ is a suitable representation of $G$ on a Banach space $E$.

3. 1 Proposition: For every $t \in G$,

$$
\sigma\left(U_{t}\right)=\left(\bigcup_{\alpha \in \operatorname{sp}(U)} \sigma\left(u_{\alpha}\left(t^{-1}\right)\right)\right)^{-}
$$

Proof (cf. [4:6.3] for the abelian case): One inclusion follows from 2.2 part.1. For the reverse inclusion suppose $\lambda \notin M:=\left(\bigcup_{a \in \operatorname{sp}(U)} \sigma\left(U_{a}\left(t^{-1}\right)\right)\right)^{-}$and let $W$ an open set in $\Gamma=\{z \in \mathbf{C}|| z \mid=1\}$ containing $M$ but $\lambda \notin \bar{W}$. We can find a $C^{2}$-function $f$ on $\Gamma$ which 
-coincides with $z \rightarrow(\lambda-z)^{-1}$ on $W$. Then the Fourier expansion is absolutely convergent and we have $f(z)=\sum_{n \in \mathbf{Z}} a_{n} z^{n}$ with $\sum_{n \in \mathbf{Z}}\left|a_{n}\right|<\infty$. Let $S=\sum_{n \in \mathbb{Z}} a_{n} U_{i n}$. In order to show $S\left(\lambda-U_{t}\right)=I$ let $\mu=\left(\sum_{n \in \mathbf{Z}} a_{n} \delta_{t n}\right) *\left(\hat{\lambda}-\delta_{l}\right)$. By 2.7 it is enough to show that $\hat{a}_{a}=I$, i.e. $\left(\sum_{n \in Z} a_{n} u_{\alpha}\left(t^{-n}\right)\right)\left(\lambda-u_{a}\left(t^{-1}\right)\right)=I$, for every $a \in \operatorname{sp}(U)$.

Let $\alpha \in \operatorname{sp}(U)$. If we consider the representation $V$ of $\mathbf{Z}$ given by $V(n)=u_{a}\left(i^{-n}\right)$, and its lifting to $l^{1}(\mathbf{Z})$ given by $V(b)=\sum_{n \in \mathbf{Z}} b_{n} u_{a}\left(t^{-n}\right)$ for $b \in l^{2}(\mathbf{Z})$ then by [4. 6.4] sp (V) $=\left[\sigma\left(u_{a}\left(t^{-1}\right)\right)\right]^{-1}$ (being aware that the co-Fourier transformation is considered in [4]). By definition of $W, \operatorname{sp}(V) \subset W^{-1}$. Consequently, $\hat{a}(z) \cdot\left(\lambda-\delta_{1}\right)^{1} \cdot(z)=f\left(z^{-1}\right)\left(\lambda-z^{-1}\right)$ $=1$ for all $z \in W^{-1}$. This implies $V(a) V\left(\lambda-\delta_{1}\right)=I$ by [4:3.3 vi], that is $\left(\sum_{n \in Z} a_{n} u_{\alpha}\left(t^{-n}\right)\right)$ $\left(\lambda-u_{\alpha}\left(t^{-1}\right)\right)=I .\left(\lambda-U_{\imath}\right) S=I$ can be shown in the same way. Hence, $\lambda \cdot \notin \sigma\left(U_{t}\right)$

For the counterexample we need some additional notation. Let $G$ be a locally compact group. For $B \subset M(G)$ we define $B^{\perp}=\{\mu \in M(G) \mid \inf \{|\mu|,|\nu|\}=0$ for all $v \in A\}$. A subset $B$ of $M(G)$ is called a band if $B=B^{11}$. If $B$ is a band in $M(G), M(G)$ is the direct sum of $B$ and $B^{1}$. By $M_{c}(G)$ we denote the space of all continuous measures in $M(G) . M_{d}(G):=M_{c}(G)$, is the space of all completely discontinuous measures in $M(G)$ and is isomorphic to $l^{1}\left(G_{d}\right) . M_{d}(G)$ is a subalgebra and $M_{c}(G)$ an algebraic ideal of $M(G)$ (see [8]). Recall, a subalgebra $A_{0}$ of an algebra $A$ is called full if for every $x \in A_{0}$, which is invertible in $A, x^{-1} \in A_{0}$.

\subsection{Lemma: $M_{d}(G)$ is a full subalgebra of $M(G)$.}

Proof: Assume $\mu \in M_{d}(G)$ is invertible in $M(G)$. We have to show that $\mu^{-1} \in M_{d}^{\prime}(G)$. There are uniquely determined ineasures $\nu_{1} \in M_{d}(G), \nu_{2} \in M_{d}(G)^{\perp}=M_{c}(G)$ such that $\mu^{-1}=\nu_{1}+\nu_{2}$. Hence $\delta_{e}=\mu * \nu_{1}+\mu * \nu_{2}$. Since $M_{c}(G)$ is an algebraic ideal, $\mu * \nu_{2}$ $=\delta_{e}-\mu * \nu_{1} \in M_{c}(G) \cap M_{d}(G)=\{0\}$. Thus $\mu * v_{2}=0$, which implies $\nu_{2}=0$, i.e. $\mu^{-1} \in M_{d}(G)$ !

Let $H$ be a subgroup of $G_{d}$ ( $G$ with the discrete topology). $l^{1}(H)$ can be identified with the band in $M(G)$ consisting of the measures in $M_{d}(G)$, which are concentrated on H.

\subsection{Lem ma: $l^{1}(H)$ is a full subalgebra of $l^{1}\left(G_{d}\right)$.}

Proof: a) $l^{1}(H)$ is a subalgebra of $l^{1}(G)$. This is obvious. b) $l^{1}(H) * l^{1}(H)^{1} \subset l^{1}(H)^{1}$ : Let $\mu \in l^{1}(H)^{\perp}$. It is enough to show that $\delta_{t} * \mu \in l^{1}(H)^{\perp}$ for all $t \in H$. Let $t \in H$. $\mu$ can be written, $\mu=\sum_{n \in \mathbb{N}} a_{n} \delta_{t_{n}}$, where $t_{n} \in G \backslash H$ and $\sum_{n \in \mathbb{N}}\left|a_{n}\right|<\infty$. Thus $\delta_{t} * \mu=\sum_{n \in \mathbb{N}} a_{n} \delta_{t i_{n}}$ $\in l^{1}(H)^{\perp}$, because $t_{n} \in G \backslash H, H$ being a subgroup of $G$.

'c) $l^{1}(H)$ is full in $l^{1}(G)$ : Assume $\mu \in l^{1}(H)$ is invertivle in $l^{1}(G)$. Then $\mu^{-1}=\nu_{1}+\nu_{2}$ for uniquely determined measures $\nu_{1} \in l^{1}(H), \nu_{2} \in l^{1}(H)^{\perp}$. Hence $\delta_{e}=\mu * \nu_{1}+\mu * \nu_{2}$, and $\mu * v_{2}=\delta_{e}-\mu * \nu_{1} \in l^{1}(H) \cap l^{1}(H)^{\perp}=\{0\}$ by a) and b); which implies $v_{2}=0$, i.e. $\mu^{-1} \in l^{1}(H)$

\subsection{Corollary: $l^{1}(H)$ can be identified with a full subalgebra of $M(G)$.}

To construct the counterexample, let $G=\operatorname{SO}(3, \mathbf{R}), E=L^{1}(G)$. Define $U$ : $G \rightarrow \mathscr{L}(E)$ by $(U ; f)(s)^{\circ}=f\left(t^{-1} s\right)(s \in G)$ for all $t \in G, f \in L^{1}(G)$. Then $U(\mu) f=\mu * f$ for $\mu \in M(G), f \in L^{1}(G)$, according to the definition in section 2. For $\mu \in M(G)$,

$$
\sigma_{M(G)}(\mu)=\sigma(U(\mu))
$$


as is well known (see also [1]). The free group on two generators $H$ is a subgroupof $G_{d}$ (see [7]). In $l^{1}(H)$ there exists a hermitian measure $\mu$ such that $i \in \sigma_{l^{1}(H)}(\mu)$ ([3]). It follows from 3.4 and (3) that $i \in \sigma(U(\mu))$ : But since $\mu$ is hermitian, the matrices $\hat{\mu}_{a}$ are selfadjoint, which implies $\bigcup_{a \in G} \sigma\left(\hat{\mu}_{a}\right) \subset \mathbf{R}$.

\section{REFERENCES}

[1] Arendt, W.: On the 0-spectrum of regular operators and the spectrum of measures. Math. Z. $17 S$ (1981), $271-287$.

[2] Arveson, W.: On groups of automorphisms of operator algebras. J. Funct. Anal. 15 (1947), $217-243$.

[3] Bonic, R. A.: Symmetry in group algebras of discrete groups. Pac. J. Math. 11 (1961), 7394.

[4] Combes, F., and.C. Delaroches : Representations des groupes localement compacts et applications aux algébres d'operateurs. Asterisque 55 (1978).

[5] D'Antoni, C., Lovgo, R., and L. Zsido: A spectral mapping theorem for locally compact groups of operators. Pacific J. Math. 103 (1982), 17-24.

[6] Evars, D. E., and T. Sund : Spectral subspaces for compact actions. Preprint.

[7] GreenleaF, F :P.: Invariant means on topological groups and their applications. New York: Van Nostrand 1969.

[8] Hewitr, E., and K. A. Ross: Abstract Harmonic Analysis. Berlin-Heidelberg-New York: Springer 1963.

[9] Størmer, E.: Regular abelian Banach algebras of linear maps of operator algebras. J. Functional Anal. 37 (1980), 331-373.

Manuskripteingang: 4. 01.1982

VERFASSER:

Dr. Wolfgang ARENDT

Mathematisches Institut der Universität

D -7400 Tübingen, Auf der Morgenstelle 10

Dr. Cla ddio D'Antoni

Istituto Matematico Università dell' Aquila

L'Aquila, Italy 